\title{
Quantifying the Effects of Beam Overlap on Radiation Damage via Radiolysis Products in the In-situ Liquid (S)TEM Cell
}

Juhan Lee $^{1}$, Daniel Nicholls ${ }^{1}$, Nigel Browning ${ }^{2}$ and B. Layla Mehdi ${ }^{1}$

${ }^{1}$ University of Liverpool, Liverpool, England, United Kingdom, ${ }^{2}$ Sivananthan Laboratories, Inc., Bolingbrook, Illinois, United States

In the last decade, in-situ liquid cell (scanning) transmission electron microscopy ((S)TEM), has brought new insights and perspectives into various disciplines; for instance, nucleation and growth phenomena, electrochemical reactions, and advances in imaging of biological cells. ${ }^{1-4}$ However, despite these significant contributions, liquid-phase electron microscopy (LPEM) has several outstanding challenges. Firstly, the only well-known quantification of radiolysis products is focused on pure water, providing their rate of formation for a given flux of high-energy electrons for a static and parallel TEM illumination only. ${ }^{5-7}$ Second, these conditions are very limited and do not consider any alternative imaging modes, such as STEM or Compressive Sensing (CS). In this study, we investigated the various beam conditions for TEM, STEM and CS by employing a reaction-diffusion model and varying the spot size, dose rate, and illumination schemes. As a proof of concept study, we focused on hydrogen gas $\left(\mathrm{H}_{2}\right)$ nanobubble formation considering its importance for fundamental aspects of liquid dynamics as well as for preventing undesired damage to the experiments.

It is well known that when an electron beam interacts with a thin liquid layer, radiolysis occurs primarily in the exposed-beam zone. As a result of the localised radiolysis process, the concentrations of the chemical species will not be homogeneous throughout the cell; therefore, diffusion is expected to take place. As illustrated in Figure 1A, a diffusion zone will thus arise around the area exposed-beam zone. Depending on the characterisation scheme being used e.g. TEM or STEM imaging mode, ${ }^{8,9}$ the electron beam can then be moved to another location either by manually changing the sample position or by using a pre-set beam movement scheme (Figure 1B, C). In these cases, if the diffusion of the species from the first beam position is fast/close enough, then they will influence the total concentration of the radiolysis in the second beam position. For this aspect, a particular simulation was carried out for 4 consecutive beam pulses. As shown in Figure $2 \mathrm{~A}, \mathrm{H}_{2}$ concentration in the $1^{\text {st }}$ beam pulse overlaps with the radiolytic species generated by the $2^{\text {nd }}$ pulse creating a gradient of radiolytic species spread out across both areas.

Since this overlap occurs as a result of diffusion generated by the species between the pulses, the level of overlap is expected to be dependent on the probe separation distance. In Figure $2 \mathrm{~B}$, the $\mathrm{H}_{2}$ concentration profiles represent the changes as a function of various separation distances between consecutive pulses/beam positions. In a case of $10 \mathrm{~nm}$ probe separation, we observe the highest $\mathrm{H}_{2}$ concentration due to direct overlap of consecutive pulses. Increasing the distance to $100 \mathrm{~nm}$, we observe beam overlap creating an additional shoulder, which increases the diffusion zone and creates a new reaction mixing zone between two consecutive probe areas. As a result, the maximum concentration of $\mathrm{H}_{2}$ becomes lower as the probe distance increases (Figure 2B). Particularly, in the case of $500 \mathrm{~nm}$ probe separation, the high concentration of the reactants in exposed- beam zone do not overlap as the species have time to diffuse away minimising the probability of generating new reactant mixing zone, and therefore, a less chance for $\mathrm{H}_{2}$ bubble formation. 
In summary, we have simulated water radiolysis under various experimental imaging conditions, using a reaction-diffusion model to understand how the distribution of incoming fast electrons affects the overall reaction dynamic and $\mathrm{H}_{2}$ formation. Using these models, we can predict the distribution of radiolysis products for a range of different illumination and sub-sampling conditions and quantify the threshold condition of the radiation damage, allowing the beam to be used to create well-controlled reactive environments in the in-situ liquid cells. These controls can be applied to any liquid system (not just water) greatly increasing the number of chemical experiments that can be performed in the microscope.
A: TEM Configuration
B: STEM Configuration
C: CS configuration
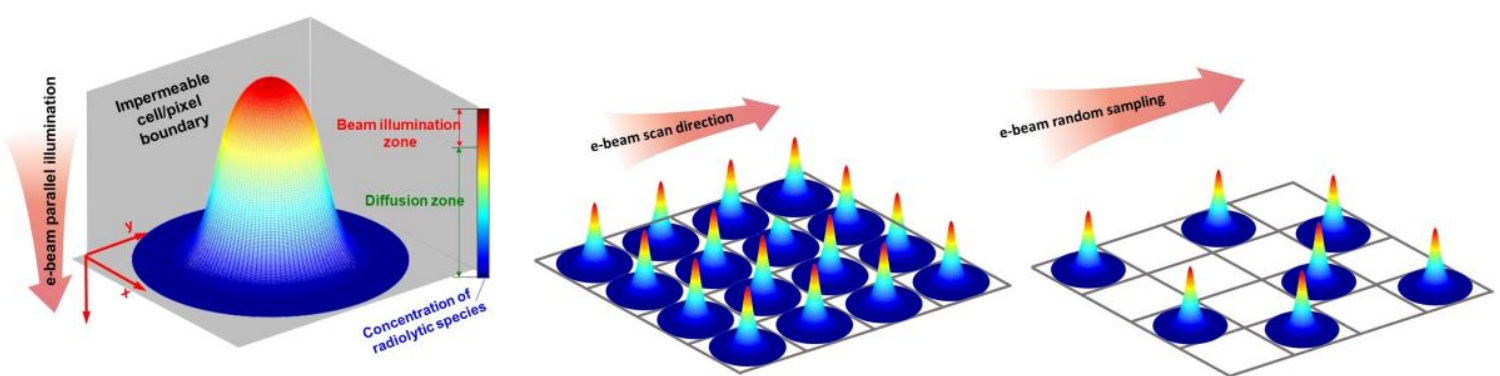

Figure 1. Schematic illustration of the e-beam interaction zones in three different imaging (A) TEM, (B) STEM and (C) random-sampling e.g. compressive sensing mode.
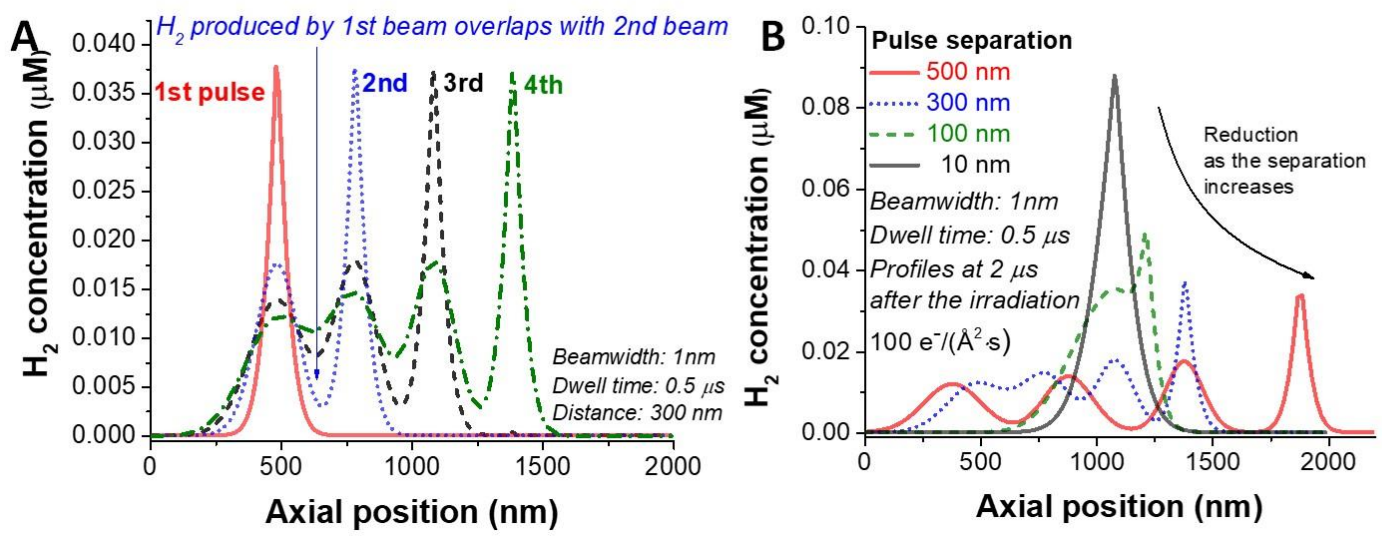

Figure 2. Influence of beam overlap for $1 \mathrm{~nm}$ probe size. (A) Spatial concentration profiles of H2 obtained for 4 probe positions with a separation distance of $300 \mathrm{~nm}$ and a dwell time of $0.5 \mu \mathrm{s}$. (B) probe separation distance in case of 10, 100, 300 and 500nm

\section{References}

1. de Jonge, N.; Ross, F. M., Electron microscopy of specimens in liquid. Nature Nanotechnology 2011, $6,695$.

2. Woehl, T. J.; Evans, J. E.; Arslan, I.; Ristenpart, W. D.; Browning, N. D., Direct in Situ Determination of the Mechanisms Controlling Nanoparticle Nucleation and Growth. ACS Nano 2012, 6, (10), 8599-8610. 3. Mehdi, B. L.; Stevens, A.; Kovarik, L.; Jiang, N.; Mehta, H.; Liyu, A.; Reehl, S.; Stanfill, B.; Luzi, L.; Hao, W.; Bramer, L.; Browning, N. D., Controlling the spatio-temporal dose distribution during STEM imaging by subsampled acquisition: In-situ observations of kinetic processes in liquids. Appl. Phys. Lett. 2019, 115, (6), 063102. 
4. Mehdi, B. L.; Stevens, A.; Qian, J.; Park, C.; Xu, W.; Henderson, W. A.; Zhang, J.-G.; Mueller, K. T.; Browning, N. D., The Impact of Li Grain Size on Coulombic Efficiency in Li Batteries. Scientific Reports 2016, 6, 34267.

5. Grogan, J. M.; Schneider, N. M.; Ross, F. M.; Bau, H. H., Bubble and Pattern Formation in Liquid Induced by an Electron Beam. Nano Lett. 2014, 14, (1), 359-364.

6. Schneider, N. M.; Norton, M. M.; Mendel, B. J.; Grogan, J. M.; Ross, F. M.; Bau, H. H., ElectronWater Interactions and Implications for Liquid Cell Electron Microscopy. The Journal of Physical Chemistry C 2014, 118, (38), 22373-22382.

7. Wang, C.; Shokuhfar, T.; Klie, R. F., Precise In Situ Modulation of Local Liquid Chemistry via Electron Irradiation in Nanoreactors Based on Graphene Liquid Cells. Adv. Mater. 2016, 28, (35), 7716-7722.

8. Downing, K. H., Spot-Scan Imaging in Transmission Electron Microscopy. Science 1991, 251, (4989), 53-59.

9. Browning, N. D.; Buban, J. P.; Chi, M.; Gipson, B.; Herrera, M.; Masiel, D. J.; Mehraeen, S.; Morgan, D. G.; Okamoto, N. L.; Ramasse, Q. M.; Reed, B. W.; Stahlberg, H., The Application of Scanning Transmission Electron Microscopy (STEM) to the Study of Nanoscale Systems. In Modeling Nanoscale Imaging in Electron Microscopy, Vogt, T.; Dahmen, W.; Binev, P., Eds. Springer US: Boston, MA, 2012; pp 11-40. 